\title{
MicroscopyEducation
}

\section{An Interactive Online Electron Microscopy Platform Integrating Classroom Lectures and Lab Practice}

\author{
Noah Freed, Devon Butler, Allison Cairns, Rick Hugo, Jun Jiao, and \\ Zhiqiang Chen*
}

Center for Electron Microscopy and Nanofabrication, Portland State University, 1025 SW Mill

St., Portland, OR 97201

*zhiqiang@pdx.edu

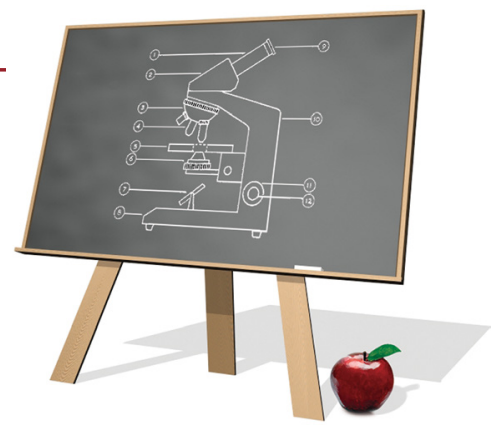

\begin{abstract}
The Online Electron Microscopy Platform makes electron microscopy education accessible by combining simulation-based practice with a range of educational content. Students learn how to operate electron microscopes by using virtual microscopes that precisely simulate the functions of real microscopes. Built as a web application, the platform can be used by an unlimited number of people and at any time. This platform reduces the time needed for training users to operate a real microscope. By preparing students in STEM disciplines to use electron microscopes, the system helps them acquire the skills they need to succeed in the modern workforce.
\end{abstract}

Keywords: electron microscope, simulator, online education, training, microscope operation

\section{Introduction}

Electron microscopy skills are valuable in today's workforce, with increased demand anticipated in the near future. However, electron microscopy skills are difficult to acquire due to the need to train on expensive equipment under instructor supervision. Becoming an independent user of an electron microscope is a time-intensive process, in our experience requiring at least 16 hours of one-on-one training. It takes a much longer time to become an efficient user. However, a typical electron microscopy class is only able to provide each student with about 4 hours of individual hands-on training over the duration of the course. One way to provide students with more opportunities to learn is through remote operation of an electron microscope in a digital lecture theater [1]. Another approach is to make model electron microscopes available to students [2]. However, even with these approaches it is still difficult to provide students with sufficient hands-on time for them to become proficient users.

Online education has the potential to provide opportunities for students to learn without such time limits. Various online education systems emerged in the late 1990s, including MIT OpenCourseWare, Stanford Engineering Everywhere, Coursera, and edX [3-9]. However, these platforms only offer lecture videos and are not able to provide lab practice. An online simulator provides a valuable supplement to time on the actual microscope, in that it both reduces the hands-on instrument time needed as well as increases the proficiency level of the users. Inspired by aviation simulators, the Online Electron Microscopy Platform (OEMP) at Portland State University (PSU) offers interactive online electron microscopy education and training via adaptive virtual microscope (VM) simulators (Figure 1). An extensive online search found there are presently six different scanning electron microscope (SEM) VMs [10-14] and two transmission electron microscope (TEM) VMs $[15,16]$ available online. The University of Illinois at Urbana-Champaign's Virtual Microscope project provides a simple SEM simulator [12]. The Wartburg College Virtual Scanning Electron Microscope [13] provides basic instruction and an interactive learning tool displaying various parts of the microscope, it includes a guided microscope "tour," and it reproduces an electron micrograph. Another virtual SEM at the University of Virginia provides basic information through animation and videos [14].

The most complete simulator system to date is the $\mathrm{K}-12$ education outreach project called MyScope virtual SEM [11] and TEM [15]. More advanced virtual TEMs have been developed by Taiwan National Hsinchu University of Education (NHCUE) [16]. NHCUE uses new technologies in virtual reality and provides excellent graphics and electron optics.

With support from the W. M. Keck Foundation, the Center for Electron Microscopy and Nanofabrication (CEMN) at Portland State University has developed web-based electron microscopy education software: the Online Electron Microscopy Platform (OEMP). The platform includes a TEM simulator, a range of educational content, a virtual specimen database, and ways for the user to customize their learning experience. The OEMP provides students the opportunity to learn electron microscopy anytime, anywhere.

The OEMP is different from existing online microscopy education in four areas [17,18]: 1) the OEMP incorporates lectures and a materials database with laboratory practice through instrument operation simulations; 2) the database allows researchers and industrial professionals to deposit new findings; 3 ) contents can be iteratively designed, piloted, and evaluated to augment EM simulators, associated new companion curricula, and ongoing classes to validate our approach; and 4) the OEMP is designed and operated as a comprehensive education platform based on online benchmarks provided by the Institute for Higher Education Policy [19]. In short, there is no virtual electron microscopy software, platform, or system that pursues simulation-based materials characterization education like the OEMP. 


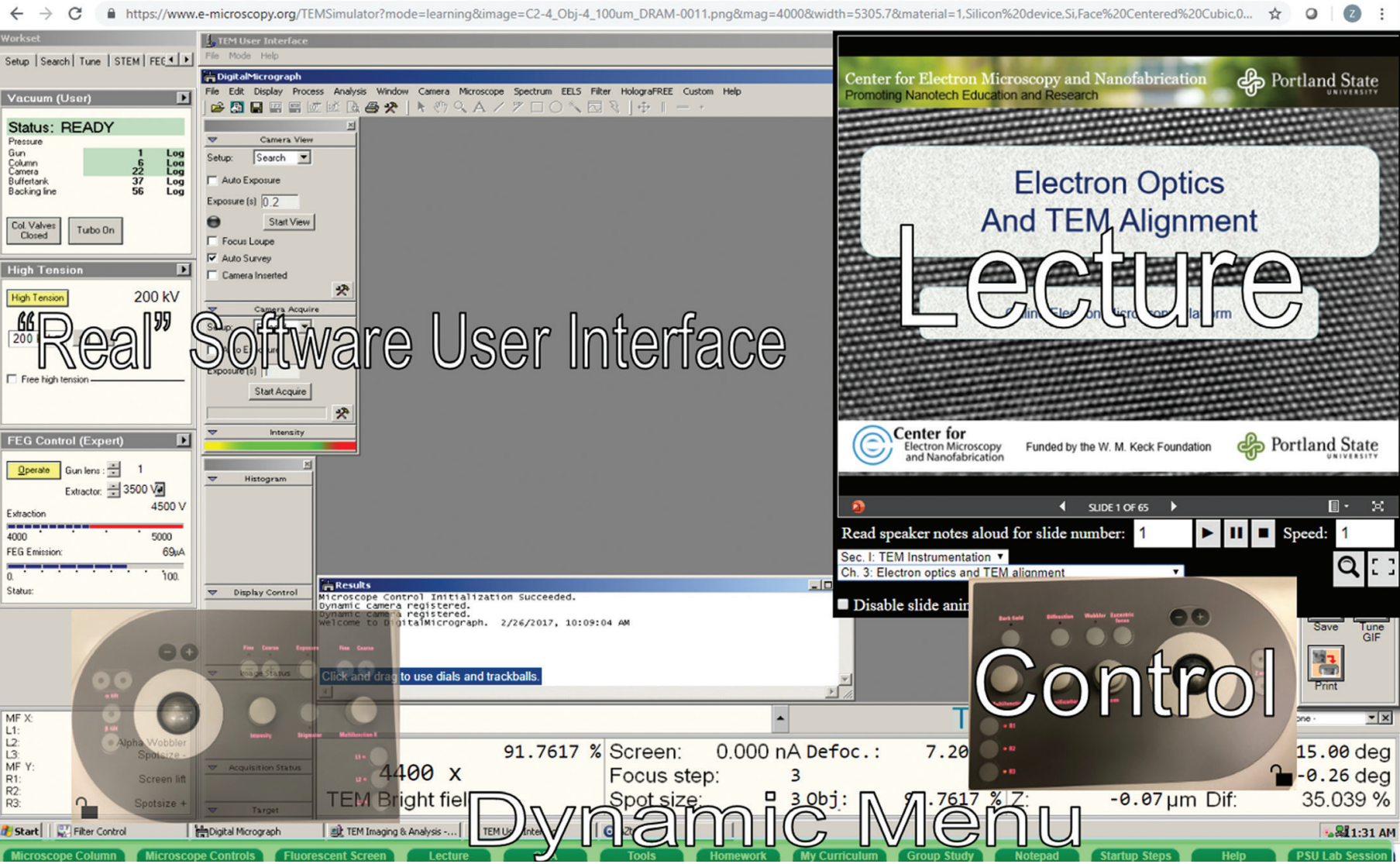

Figure 1: Image of the user interface showing the lecture content and microscope controls available. The OEMP offers high-fidelity online simulation of electron microscopes including lecture material and controls for interfacing with the virtual microscope.

\section{Electron Microscope Simulator}

At the heart of the OEMP is an electron microscope simulator designed to provide users with virtual lab practice. Other electron microscope simulators have been established, such as Microscopy Australia's SEM and TEM simulators [11,15]. These are beginner-friendly, with step-by-step guides to direct the user in operation. However, they do not allow for a full, comprehensive range of operations on the electron microscope. Users are also forced to operate the VM with guidance, and independent practice of instrument setup is not allowed. In contrast, the OEMP VM has an unparalleled breadth of functionality, and all guidance is optional. This grants users the freedom to explore, make mistakes, and learn by doing, all without risk to any actual instrument.

The electron microscope simulator we developed is based on the FEI Tecnai F-20 TEM/STEM. The simulator includes simulated controls and a user interface that matches the real hardware and software employed in operating the TEM (Figure 1). Accompanying the interface is a visual display of the microscope column, allowing users to see the various components at work in the microscope from the electron gun, down through various apertures and lenses, all the way to the viewing screen. Users can also view the fluorescent screen itself to see the virtual specimen they are working with (Figure 2).

The simulator is capable of multiple modes of operation. These modes include imaging, diffraction, scanning, energy-dispersive X-ray spectroscopy (EDX), electron energy loss spectroscopy (EELS), and energy-filtered transmission electron microscopy (EFTEM). Information provided through the VM is based on the virtual specimen and mode chosen by the user. For example, in the imaging mode, users see a highresolution picture of the specimen, while in the diffraction mode users see a dot pattern based on the crystal structure of the element at the center of the electron beam.

Via the on-screen controls, users can perform several functions to adjust the image seen. These functions include beam shift, stage movement, and direct alignments. Users can adjust magnification, focus, intensity, apertures, stigmators, and spot size. Each manipulation is performed with simulated knobs, buttons, and trackballs that mirror actual control design.

\section{The Online Electron Microscopy Platform (OEMP)}

The OEMP is a website that uniquely integrates electron microscope simulators with more traditional classroom content, such as lectures and homework. There are no limits on how many users may simultaneously access the OEMP. From the main page (Figure 3), users can customize their syllabus, select a virtual specimen to view in the simulator, resume using the simulator with their last selected specimen, or contribute data to the platform. Currently students may only access the TEM simulator, but other simulators are planned for future development. 




Figure 2: The OEMP incorporates theoretical lectures, a specimen database, and a virtual fluorescent screen for laboratory practice.

Contributed data allow for expanding the platform. Users can submit information about different types of specimens, images of specimens, and spectral data for use in the microscope simulator. Instructors may also submit their own presentations to expand the lecture content available for students.

Users may also select a syllabus based on subject matter (such as Physics or Biology), or fully customize their own syllabus on a chapter-by-chapter basis (Figure 4). Users may then select a simulator mode (learning, introduction, or self-assessment) and specimen image to use in the simulator (Figure 5). The selected mode determines the level of guidance, with the introduction mode including step-by-step instructions, the learning mode providing supplemental information on specific tasks, and the self-assessment mode acting as a means for users to test their own knowledge. Over 60 users have used the OEMP. It has been used in a TEM class at PSU, as well as by independent students seeking training outside of a standard class setting.

\section{Learning Environment of the OEMP}

A variety of interactive educational content is integrated with the simulator. This content includes:

- Lectures: Features searchable presentations with audio notes and embedded content, including videos and adjustable graphs.

- Q\&A: Users can search previously answered questions or submit new questions.
- Tools: Provides interactive animations, demonstrations, and applications designed to improve student understanding.

- Homework: Provides multiple-choice quizzes on the lecture material.

- My Curriculum: Provides a selected syllabus for the current course of study.

- Group Study: Provides a collection of chat rooms to support student communication with each other and with instructors.

- Notepad: Provides an area for users to take notes.

Also included are general help windows and other guidance, such as start-up steps and alignment help. When a user attempts to perform an action that could damage a real microscope, an error message appears explaining the risks and guiding the user on how to safely proceed.

The user interface is adjustable, with users able to freely open, close, move, and resize the windows associated with each piece of educational content. Users can also show or hide the microscope controls and apertures and can move them around the screen or lock them in place. This lets each user adjust their learning environment to best meet their needs and educational goals.

\section{Classroom Evaluation Method and Results}

To test the effectiveness of the OEMP as an educational tool, we made it available to students in a TEM class at PSU. We tracked usage and progress in the simulator and surveyed students on their experience using the OEMP (Figure 6). Students considered the OEMP to be helpful, with one stating that "the 


\section{Online Electron Microscopy Platform}

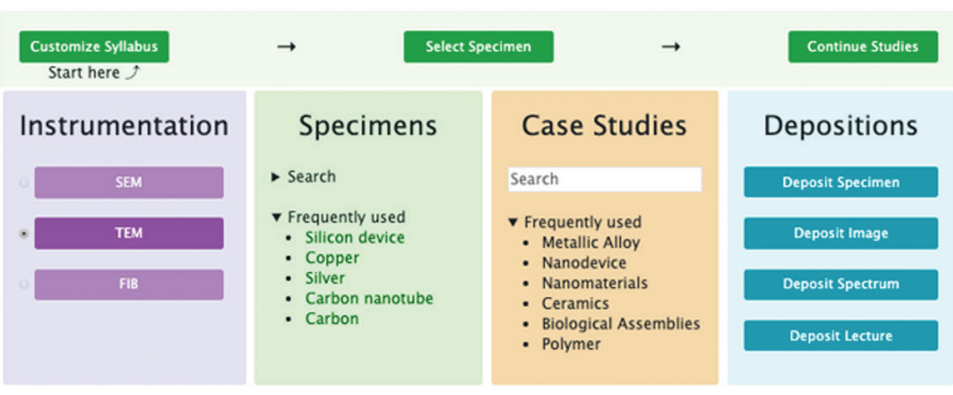

You are currently logged in as zhiqiang.

Logout

Please use Google Chrome on a desktop computer when exploring the Online Electron Microscopy Platform.

Terms and Conditions

For additional support, please contact cemn@pdx.edu.

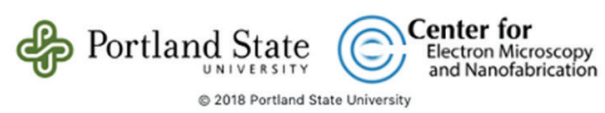

Figure 3: The main page of the Online Electron Microscopy Platform illustrating the process for accessing a simulator and options available for the type of instrument, deposited specimens and case studies, and pages for adding specimen information to the database.

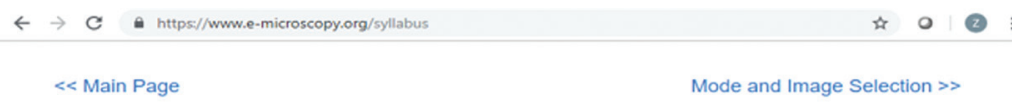

TEM Syllabus

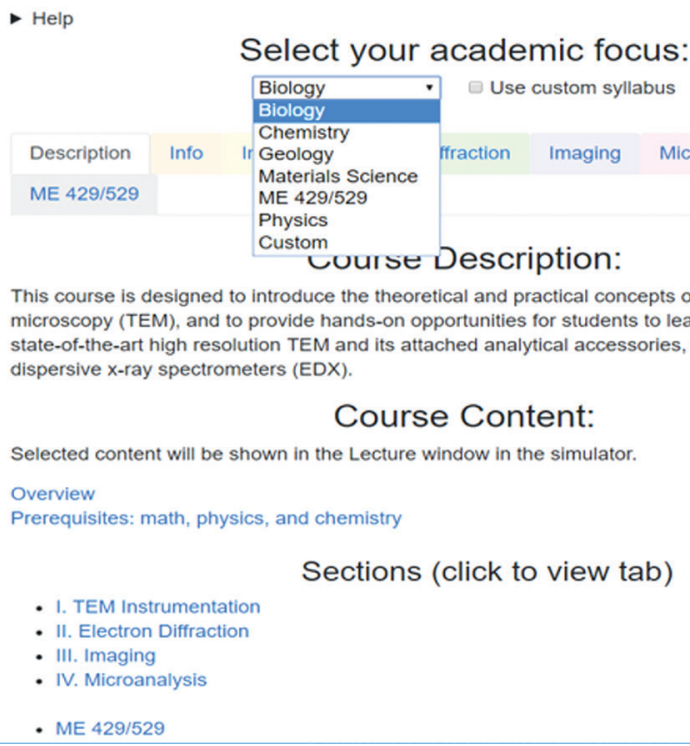

Figure 4: An image from the syllabus page for the TEM course showing some of the options available for study. By selecting the appropriate option, users can adjust their academic focus. simulator greatly improved my learning outcome." We also found a positive correlation between OEMP use and the scores students received on assignments and exams in the TEM class (Figure 7).

\section{Challenges and Potential Solutions}

While the OEMP is a useful tool for teaching students electron microscopy, we did encounter some challenges that should be considered. These challenges are common to many forms of online education.

Student adaptability: Switching from conventional classroom and face-to-face instructor lab training to web-based training in the simulator makes the learning experience entirely different for students. For some students, their resistance to change makes it difficult for them to adapt to the online learning environment if they also have an option for a traditional classroom and lab. Students with a "traditional" mindset find it difficult to adapt to online training opportunities.

Student time management and self-motivation: Self-motivation is an essential requirement for online learning, however, many students lack it. Some students take the class because it is a required class for their degree. These students learn simply to pass the class. It is a challenge to help these students find the motivation to follow the online education program and to also properly equip themselves for future challenges in their education and careers.

We hope to address these challenges through various means. Solutions take the form of changes to the platform or additional programs built around the platform. Potential approaches we are considering include:

- Offering workshops to help bridge the gap between in-person and online learning,

- Incorporating additional feedback and progress tracking into the platform,

- Creating discrete problem sets for learning how to apply different techniques, and

- Developing a certification program to acknowledge and reward mastery of electron microscopy techniques.

By augmenting the platform and introducing new programs to maximize student engagement, we intend to help students across STEM disciplines learn electron microscopy.

\section{Conclusion and Future Development}

The OEMP has been developed successfully to integrate conventional classroom learning with high-fidelity lab practice. The OEMP offers lecture presentations, quizzes, Q\&A, group study, and multiple learning paths. The platform allows students to customize their own syllabus and learning objectives. The site supports dynamic content updates by user and instructor submissions.

Planned future development includes improved support for multi-element specimens; simulators 


\section{Mode and Image Selection}

\section{Choose which mode and image you wish to use in the electron microscope simulator}

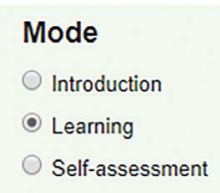

\section{Nanodevice}

Diode

Bipolar Junction Transistor

CMOS

NAND

- DRAM

SRAM

Carbon nanotube

Carbon

Ti6Al4V

Crossgrating $\$ 106$

\section{Images}

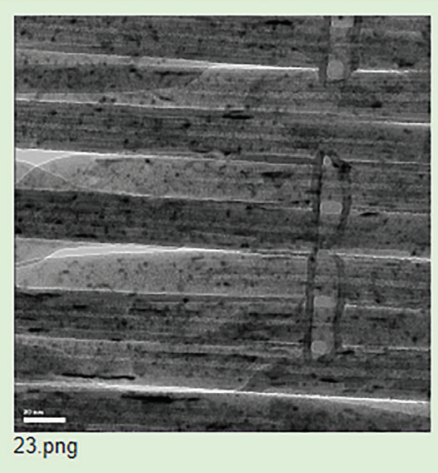

Tony Chen

CEMN at PSU

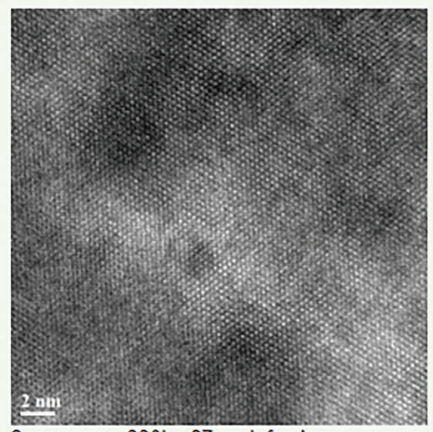

3 sec-expos-880kx-67nmdefoc.jpg

Wayde Harris

Portland State University Center for Electron Microscopy and Nanofabrication

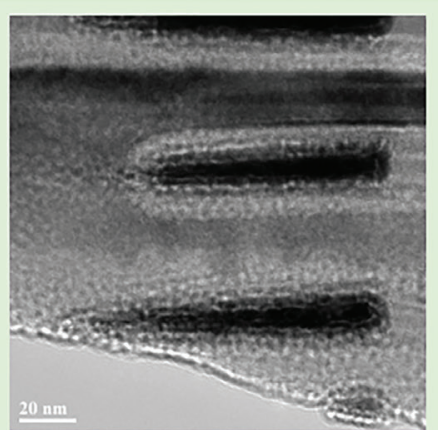

01_VeryOverfocusedDRAM-0017.png

Wayde Harris

Portland State University Center for

Electron Microscopy and Nanofabrication



02_SlightOverfocusDRAM-0016.png

Wayde Harris

Portland State University Center for Electron Microscopy and Nanofabrication

Figure 5: From the Mode and Image Selection page, each user can select from introduction, learning, or self-assessment modes and the image to be used during simulation.

\begin{tabular}{|l|l|c|}
\hline \# & \multicolumn{1}{|c}{ Question } & Survey Score \\
\hline $\mathbf{1}$ & The software was easy to use. & $3.56 / 5$ \\
$\mathbf{2}$ & $\begin{array}{l}\text { The software was free of bugs. } \\
\text { The interactive TEM simulation helped me learn } \\
\text { practical TEM skills }\end{array}$ & $3.00 / 5$ \\
\hline $\mathbf{4}$ & $\begin{array}{l}\text { The software knowledge base helped me learn practical } \\
\text { TEM skills. }\end{array}$ & $3.81 / 5$ \\
\hline $\mathbf{5}$ & $\begin{array}{l}\text { The interactive TEM simulation helped me understand } \\
\text { TEM theory. }\end{array}$ & $3.81 / 5$ \\
\hline $\mathbf{6}$ & $\begin{array}{l}\text { The software knowledge base helped me understand } \\
\text { TEM theory. }\end{array}$ & $3.25 / 5$ \\
\hline $\mathbf{7}$ & $\begin{array}{l}\text { The embedded quizzes helped me to understand the } \\
\text { material in the OEMP software knowledge base. }\end{array}$ & $3.88 / 5$ \\
\hline $\mathbf{8}$ & $\begin{array}{l}\text { The OEMP helped me to complete my TEM laboratory } \\
\text { assignments and reports. }\end{array}$ & $2.93 / 5$ \\
\hline $\mathbf{9}$ & $\begin{array}{l}\text { The OEMP helped me to complete my TEM textbook } \\
\text { assignments and quizzes. }\end{array}$ & $3.88 / 5$ \\
\hline & $3.67 / 5$ \\
\hline
\end{tabular}

Figure 6: In-class assessment scores showing that the OEMP improved practical skills and helped students understand TEM theory. for other instruments, such as a SEM and a focused ion beam instrument; and problem sets, where students perform experiments using specified techniques. A long-term goal for the OEMP is to produce a generalizable program structure that can be adapted broadly to support different types of instrument simulators. Users from other institutions will be able to contribute to the platform by helping to develop simulators for their areas of interest, or by contributing content to our virtual specimen and problem set database.

\section{Acknowledgements}

The authors would like to acknowledge funding from the W. M. Keck Foundation and thank ThermoFisher Scientific (formerly FEI), Oxford Instruments, and Gatan for their support. ThermoFisher, Oxford Instruments, and Gatan allowed us to use their software user interface designs in developing the TEM simulator, and ThermoFisher provided text and images to be used for help documents in the simulator. 
Effect of OEMP use on TEM assignment and exam grades

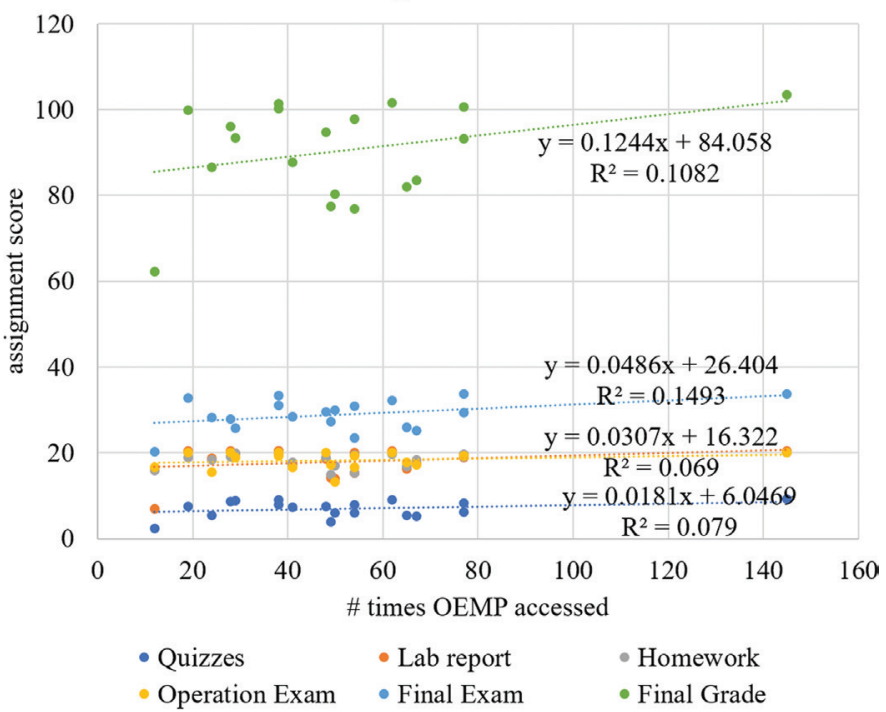

Figure 7: A positive correlation exists between the number of times students accessed the OEMP and their scores on class assignments.

\section{References}

[1] DE Huber et al., Microscopy Today 26 (2018) 26.

[2] NV Hudson-Smith et al., J Chem Education 96 (2019) 1377-82.

[3] Stanford University, "Engineering Everywhere," www.eie. org/engineeringeverywhere.
[4] MIT OpenCourseWare, https://ocw.mit.edu/index.htm.

[5] UC Berkeley edX, www.edx.org.

[6] Coursera, www.coursera.org.

[7] nanoHUB, https://nanohub.org/resources/4092.

[8] Carnegie Mellon University, "Open Learning Initiative," http://oli.cmu.edu.

[9] Yale University, “Open Yale Courses," https://oyc.yale.edu/courses.

[10] Florida State University, "Virtual Scanning Electron Microscopy," http://micro.magnet.fsu.edu/primer/java/ electronmicroscopy/magnifyl.

[11] "MyScope OutReach, Explore with the SEM Simulator," http://myscopeoutreach.org/virtualSEM_explore.html.

[12] University of Illinois at Urbana-Champaign, "The Virtual Microscope," http://virtual.itg.uiuc.edu.

[13] Wartburg College Biology Department, "Virtual: Scanning Electron Microscope," http://vip.wartburg.edu/biology/SEMweb/index.htm.

[14] Virtual Lab, "Scanning Electron Microscope (SEM)," https://wecanfigurethisout.org/VL/SEM.htm/state/1.

[15] MyScope, "Virtual TEM - Diffraction and advanced imaging," https:/myscope.training/legacy/tem/practice/ virtualtem/advanced.php.

[16] W Tarng et al., Virtual Reality 19(1) (2015) 33-44.

[17] Online Electron Microscopy Platform, www.e-microscopy.org.

[18] N Freed et al., Microsc Microanal 25 (Suppl 2) (2019) 2296-97.

[19] The Institute for Higher Education Policy, "Quality on the Line: Benchmarks for success in Internet-based Distance Education," National Education Association (2000) 1-32.

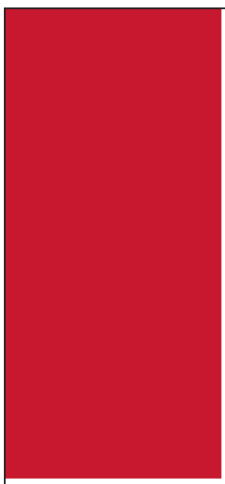

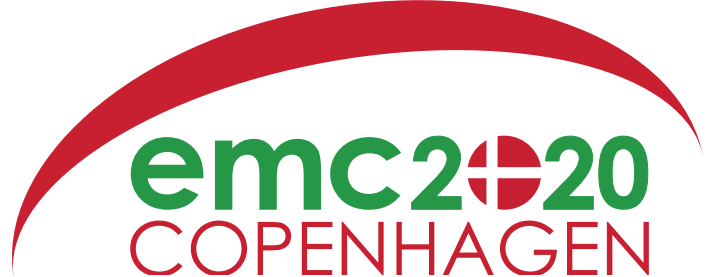

EUROPEAN MICROSCOPY CONGRESS 2020

23 - 28 August 2020, Copenhagen, Denmark

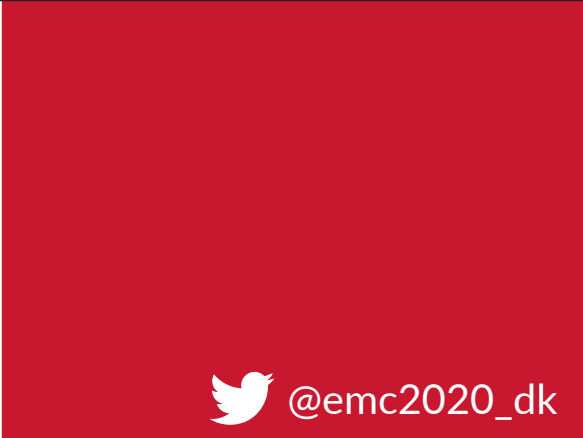

\section{Europe's Largest Event Dedicated to Microscopy and Imaging}

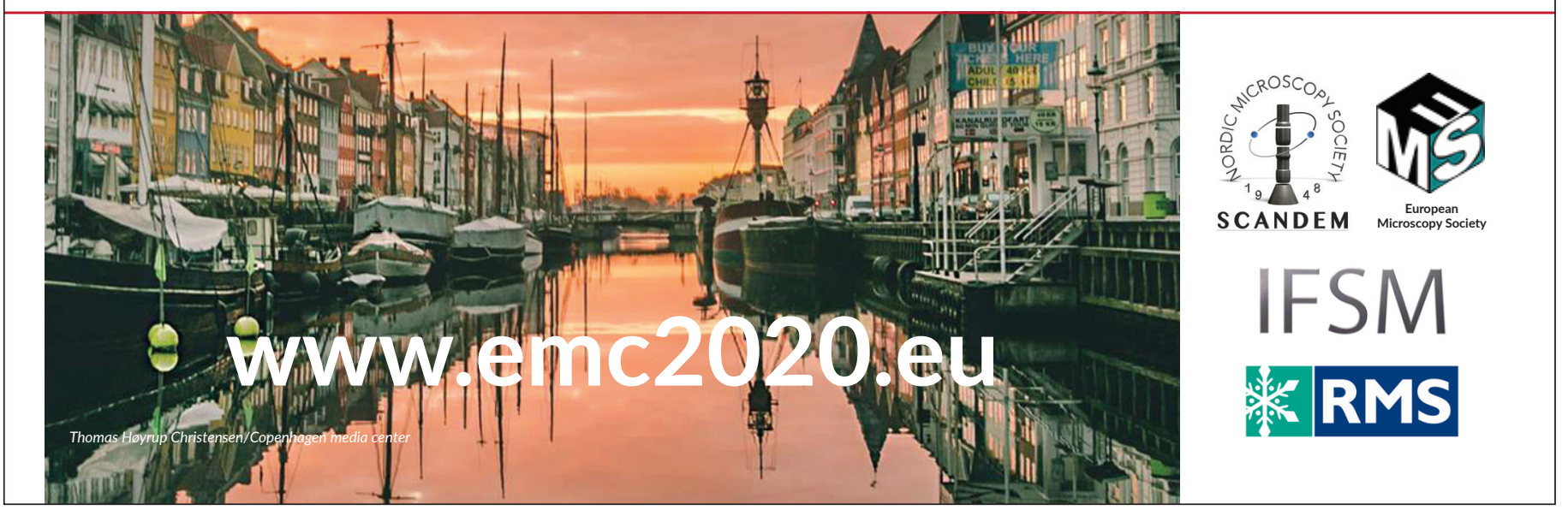

